\title{
Fractional Euler Lagrange Equations for Irregular Lagrangian with Holonomic Constraints
}

\author{
Ola A. Jarab'ah \\ Applied Physics Department, Faculty of Science, Tafila Technical University, Tafila, Jordan \\ Email: oasj85@yahoo.com
}

How to cite this paper: Jarab'ah, O.A. (2018) Fractional Euler Lagrange Equations for Irregular Lagrangian with Holonomic Constraints. Journal of Modern Physics, 9, 1690-1696.

https://doi.org/10.4236/jmp.2018.98105

Received: July 10, 2018

Accepted: July 28, 2018

Published: July 31, 2018

Copyright $\odot 2018$ by author and Scientific Research Publishing Inc. This work is licensed under the Creative Commons Attribution International License (CC BY 4.0).

http://creativecommons.org/licenses/by/4.0/

\begin{abstract}
In this paper the fractional Euler Lagrange equations for irregular Lagrangian with holonomic constraints have been presented. The equations of motion are obtained using fractional Euler Lagrange equations in a similar manner to the usual mechanics. The results of fractional calculus reduce to those obtained from classical calculus (the standard Euler Lagrange equations) when $\gamma \rightarrow 0$ and $\alpha, \beta$ are equal unity only. Two problems are considered to demonstrate the application of the formalism.
\end{abstract}

\section{Keywords}

Fractional Derivatives, Euler-Lagrange Equations, Irregular Lagrangian, Holonomic Constraints

\section{Introduction}

The Euler Lagrange equations and Hamilton's principle form the basis of Lagrangian or Hamiltonian mechanics. The power of Lagrangian mechanics is that the given equations are characterized with only one scalar function the Lagrangian L, or the Hamiltonian $\mathrm{H}$. In general, these functions only describe conservative systems. There have been some approaches at describing nonconservative systems in such formalism. The method presented by Rayleigh introduces a function R (called Rayleigh's dissipation function).

The study of holonomic constrained systems is discussed in most references of classical mechanics [1] [2]; these systems describe dynamic systems with constraints depend only on the generalized coordinates $q_{i}(t)$. The canonical formalism of holonomic systems was treated by Rabei [3]. In this formalism the 
author has treated the regular Lagrangian with holonomic constraints as singular systems and the Lagrange multipliers as generalized coordinates. The equations of motion are written as total differential equations then, the holonomic systems are quantized using the WKB approximation [4].

Fractional calculus is a generalization of differentiation and integration to a noninteger order. The interest in fractional calculus has been growing continually during the last few years because of its numerous applications in science and engineering [5] [6] [7]. The traditional calculus of variation cannot be used to obtain the equations of motion for the nonconservative systems.

Riewe has used the fractional calculus to develop a formalism which can be used for both conservative and nonconservative systems [8] [9]. The Hamiltonian and Lagrangian involving fractional derivative is also used to derive the equation of damped harmonic oscillator [10]. Therefore the dynamical systems with fractional order can be dissipative. For this reason, the theory and methods of fractional calculus are extensively used for describing critical phenomena in nonconservative systems of physics and mechanics [11] [12].

Recently, the classical calculus of variations was extended by Agrawal [13] for systems containing Riemann-Liouville fractional derivatives. The resulting equations are found to be similar to those for variational problems containing integral order derivatives. In other words, the results of fractional calculus of variations reduce to those obtained from traditional fractional calculus of variations when the derivative of fractional order replaced by integral order. More recently, Euler Lagrange equations for holonomic constrained systems with regular Lagrangian have been presented by Hasan [14] using the fractional variationl problems.

In the present paper as a continuation of Jarab'ah work [15] the fractional Euler Lagrange equations are used to obtain the equations of motion for irregular Lagrangian with holonomic constraints, it seems that there are several choices of fractional Lagrangian giving the same classical limit, in other words the same classical Lagrangian.

This paper is organized as follows: In Section 2, Euler Lagrange equations formulation for Irregular Lagrangian with holonomic constraints is reviewed briefly. In Section 3, basic definitions of fractional derivatives are briefly discussed. In Section 4, the fractional Euler Lagrange equations for irregular Lagrangian with holonomic constraints are explained. In Section 5, two illustrative examples are examined. The work closes with some concluding remarks (In Section 6).

\section{Euler Lagrange Equations Formulation for Regular Lagrangian with Holonomic Constraints}

The Lagrangian formulation for regular Lagrangian is given by

$$
L_{\circ}=L_{\circ}\left(q_{i}, \dot{q}_{i}, t\right)
$$

Here $L_{\circ}\left(q_{i}, \dot{q}_{i}, t\right)$ stands for the Lagrangian of the corresponding conserva- 
tive systems. The standard method for incorporating the constraint functions to the equations of motion is the use of the so called Lagrange multipliers. The motion of a holonomic system could in principle be determined by making use of the $n$ Euler Lagrange equations and $m$ constraints.

$$
\frac{\mathrm{d}}{\mathrm{d} t}\left(\frac{\partial L_{\circ}}{\partial \dot{q}_{i}}\right)-\frac{\partial L_{\circ}}{\partial q_{i}}=\lambda_{\mu} \frac{\partial f_{\mu}}{\partial q_{i}}, i=1,2, \cdots, n
$$

The constraint equation with $m$ constraints can be written as $f_{\mu}\left(q_{i}, t\right)=0$, $\mu=n+1, n+2, \cdots, n+m$.

$\lambda_{\mu}$ : are the Lagrange multipliers.

\section{Basic Definitions of Fractional Derivatives}

Now, we will give the basic definition of a fractional derivatives include the left and the right Riemann Liouville fractional derivatives [16] [17] and their properties. The left Riemann Liouville fractional derivative is defined as

$$
{ }_{a} D_{x}^{\alpha} f(x)=\frac{1}{\Gamma(n-\alpha)}\left(\frac{\mathrm{d}}{\mathrm{d} x}\right)^{n} \int_{a}^{x}(x-\tau)^{n-\alpha-1} f(\tau) \mathrm{d} \tau
$$

which is denoted as the LRLFD,

and the right Riemann Liouville fractional derivative is defined as

$$
{ }_{x} D_{b}^{\alpha} f(x)=\frac{1}{\Gamma(n-\alpha)}\left(-\frac{\mathrm{d}}{\mathrm{d} x}\right)^{n} \int_{x}^{b}(\tau-x)^{n-\alpha-1} f(\tau) \mathrm{d} \tau
$$

which is denoted as the RRLFD,

Here $\Gamma$ represents the Euler's gamma function, and $\alpha$ is the order of the derivative such that $n-1 \leq \alpha<n$ and is not equal to zero. If $\alpha$ is an integer, these derivatives are defined as follows:

$$
\begin{aligned}
{ }_{a} D_{x}^{\alpha} f(x) & =\left(\frac{\mathrm{d}}{\mathrm{d} x}\right)^{\alpha} f(x) \\
{ }_{x} D_{b}^{\alpha} f(x) & =\left(-\frac{\mathrm{d}}{\mathrm{d} x}\right)^{\alpha} f(x) \\
\alpha & =1,2, \cdots
\end{aligned}
$$

The fractional operator ${ }_{a} D_{x}^{\alpha} f(x)$ can be written as [18].

$$
{ }_{a} D_{x}^{\alpha}=\frac{\mathrm{d}^{n}}{\mathrm{~d} x^{n}}{ }_{a} D_{x}^{\alpha-n}
$$

and has the following properties:
1) ${ }_{a} D_{x}^{\alpha}=\frac{\mathrm{d}^{\alpha}}{\mathrm{d} x^{\alpha}}, \quad \operatorname{Re}(\alpha)>0$
2) ${ }_{a} D_{x}^{\alpha}=1, \quad \operatorname{Re}(\alpha)=0$
3) ${ }_{a} D_{x}^{\alpha}=\int_{a}^{x}(\mathrm{~d} \tau)^{-\alpha}, \quad \operatorname{Re}(\alpha)<0$

Theorem: Let $f$ and $g$ be two continuous functions on $[a, b]$. Then, for all 
$x \in[a, b]$, the following properties hold:

1) For $m>0,{ }_{a} D_{x}^{m}[f(x)+g(x)]={ }_{a} D_{x}^{m} f(x)+{ }_{a} D_{x}^{m} g(x)$

2) For $m \geq n \geq 0,{ }_{a} D_{x}^{m}\left({ }_{a} D_{x}^{-n} f(x)\right)={ }_{a} D_{x}^{m-n} f(x)$

3) For $m>0,{ }_{a} D_{x}^{m}\left({ }_{a} D_{x}^{-m} f(x)\right)=f(x)$

4) For $m>0, \int_{a}^{b}\left({ }_{a} D_{x}^{m} f(x)\right) g(x) \mathrm{d} x=\int_{a}^{b} f(x)\left({ }_{x} D_{b}^{m} g(x)\right) \mathrm{d} x$

\section{Fractional Euler Lagrange Equations for Irregular Lagrangian with Holonomic Constraints}

The Lagrangian formulation for irregular Lagrangian without fractional derivatives is given by [19]

$$
L=L_{\circ}\left(q_{i}, \dot{q}_{i}\right) \mathrm{e}^{\gamma t}
$$

where $L$ is irregular Lagrangian which is a function of $n$ generalized coordinates $q_{i}(t)$ and $n$ generalized velocities $\dot{q}_{i}(t)$ and $\gamma$ is defined as damping factor.

The Euler-Lagrange equation for the fractional calculus of variations problems is obtained as

$$
\frac{\partial L}{\partial q}+{ }_{t} D_{b}^{\alpha} \frac{\partial L}{\partial_{a} D_{t}^{\alpha} q}+{ }_{a} D_{t}^{\beta} \frac{\partial L}{\partial_{t} D_{b}^{\beta} q}+\lambda_{\mu} \frac{\partial f}{\partial q}=0
$$

Here $L$ is a function of the form $L=L_{\circ}\left(q,{ }_{a} D_{t}^{\alpha} q, D_{t}^{\beta} q\right) \mathrm{e}^{\gamma t}$.

It is worth to mention that for $\alpha=\beta=1$, we have ${ }_{a} D_{t}^{\alpha}=\frac{\mathrm{d}}{\mathrm{d} t}$ and

${ }_{t} D_{b}^{\alpha}=-\frac{\mathrm{d}}{\mathrm{d} t}$ and Equation (9) reduces to the standard Euler Lagrange equation for holonomic constraints.

\section{Examples}

1) As a first model let us consider the following Lagrangian that describes the motion of a bead of mass $m$ is constrained to move on a circular wire of radius $R$.

The Lagrangian of our problem is given by

$$
L=\frac{1}{2} m\left(\dot{r}^{2}+r^{2} \dot{\theta}^{2}\right)-m g r \cos \theta
$$

In the presence of damping factor the Lagrangian becomes

$$
L=\left[\frac{1}{2} m\left(\dot{r}^{2}+r^{2} \dot{\theta}^{2}\right)-m g r \cos \theta\right] \mathrm{e}^{\gamma t}
$$

Is subject to the holonomic constraint

$$
f(r)=r-R=0
$$

The Lagrangian in fractional form can be written as

$$
L=\left[\frac{1}{2} m\left({ }_{0} D_{t}^{\alpha} r\right)^{2}+\frac{1}{2} m r^{2}\left({ }_{t} D_{1}^{\beta} \theta\right)^{2}-m g r \cos \theta\right] \mathrm{e}^{\gamma t}
$$


Using Equation (9), the corresponding Euler Lagrange equations are

$$
\mathrm{e}^{\gamma t} m r\left({ }_{t} D_{1}^{\beta} \theta\right)^{2}-\mathrm{e}^{\gamma t} m g \cos \theta+{ }_{t} D_{1}^{\alpha}\left[m\left({ }_{0} D_{t}^{\alpha} r\right) \mathrm{e}^{\gamma t}\right]+\lambda=0
$$

and

$$
\mathrm{e}^{\gamma t} m g r \sin \theta+{ }_{0} D_{t}^{\beta}\left[m r^{2}\left({ }_{t} D_{1}^{\beta} \theta\right) \mathrm{e}^{\gamma t}\right]=0
$$

From Equations (13) and (14), we obtain the classical results if $\gamma \rightarrow 0$ and $\alpha, \beta=1$. One can get the angular acceleration

$$
\ddot{\theta}=\frac{g \sin \theta}{R}
$$

and the Lagrange multiplier is given by

$$
\lambda=m g(3 \cos \theta-2)
$$

2) As a second model let us consider the following Lagrangian that describes the motion of a disk of mass $m$ and radius $R$ that is rolling down an inclined plane without slipping.

The Lagrangian of our problem is given by

$$
L=\frac{1}{2} m \dot{y}^{2}+\frac{1}{4} m R^{2} \dot{\theta}^{2}+m g y \sin \varphi
$$

In the presence of damping factor the Lagrangian becomes

$$
L=\left[\frac{1}{2} m \dot{y}^{2}+\frac{1}{4} m R^{2} \dot{\theta}^{2}+m g y \sin \varphi\right] \mathrm{e}^{\gamma t}
$$

where $\varphi$ is the angle of the inline plane.

The holonomic constraint equation is

$$
f(y, \theta)=y-R \theta=0
$$

The Lagrangian in fractional form can be written as

$$
L=\left[\frac{1}{2} m\left({ }_{0} D_{t}^{\alpha} y\right)^{2}+\frac{1}{4} m R^{2}\left({ }_{t} D_{1}^{\beta} \theta\right)^{2}+m g y \sin \varphi\right] \mathrm{e}^{\gamma t}
$$

Using Equations (9), the corresponding Euler Lagrange equations are

$$
\mathrm{e}^{\gamma t} m g \sin \varphi+{ }_{t} D_{1}^{\alpha}\left[m\left({ }_{0} D_{t}^{\alpha} y\right) \mathrm{e}^{\gamma t}\right]+\lambda=0
$$

and

$$
\frac{1}{2} m R_{0}^{2} D_{t}^{\beta}\left[\left({ }_{t} D_{1}^{\beta} \theta\right) \mathrm{e}^{\gamma t}\right]-R \lambda=0
$$

again, making use of Equations (20) and (21), if $\gamma \rightarrow 0$ and $\alpha, \beta=1$. The angular accelerations take the following form

$$
\begin{gathered}
\ddot{\theta}=\frac{2}{3} \frac{g}{R} \sin \varphi \\
\ddot{y}=\frac{2}{3} g \sin \varphi
\end{gathered}
$$

and the Lagrange multiplier 


$$
\lambda=\frac{-1}{3} m g \sin \varphi
$$

which are in exact agreement with that obtained by classical method.

\section{Conclusion}

In this work, Euler Lagrange equations have been presented for irregular Lagrangian with holonomic constraints using fractional approach, the fractional Euler Lagrange equations for holonomic constrained systems were derived, and through this approach we have shown that, the fractional results are very similar to those for the classical results. In special cases, when $\gamma \rightarrow 0$ and $\alpha, \beta$ are equal unity only; the results of fractional calculus reduce to those obtained from classical calculus. Given the fact that many systems can be modeled more accurately using fractional derivative models, it is hoped that future research will continue in this area.

\section{Conflicts of Interest}

The authors declare no conflicts of interest regarding the publication of this paper.

\section{References}

[1] Atam, A.P. (1990) Introduction to Classical Mechanics. Allyn and Bacon, Needham Heights.

[2] Goldstein, H. (1980) Classical Mechanics. 2nd Edition, Addison-Wesley, Reading.

[3] Rabei, E.M. (1999) Turkish Journal of Physics, 23, 1083. http://adsabs.harvard.edu/abs/2000NCimB.115.1159R

[4] Serhan, M., Abusini, M. and Rabei, E.M. (2009) Journal of Theoretical Physics, 48, 2731. https://doi.org/10.1007/s10773-009-0063-5

[5] Miller, K.S. and Ross, B. (1993) An Introduction to the Fractional Integrals and Derivatives-Theory and Applications. John Willey and Sons, New York.

[6] Samko, S.G., Kilbas, A.A. and Marichev, O.I. (1993) Fractional Integrals and Derivatives: Theory and Applications. Gordon and Breach Science Publishers, Amsterdam.

[7] Gorenflo, R. and Mainardi, F. (1997) Fractional Calculus: Integral and Differential Equations of Fractional Orders, Fractals and Fractional Calculus in Continuums Mechanics. Springer Verlag, Wien and New York.

[8] Riewe, F. (1996) Physical Review E, 53, 1890. https://doi.org/10.1103/PhysRevE.53.1890

[9] Riewe, F. (1997) Physical Review E, 55, 3581. https://doi.org/10.1103/PhysRevE.55.3581

[10] Tarawneh, K.M., Rabei, E.M. and Ghassib, H.B. (2010) Journal of Dynamics Systems and Theories, 8, 59-70. https://doi.org/10.1080/1726037X.2010.10698578

[11] Hilfer, R. (2000) Applications of Fractional Calculus in Physics. World Scientific Publishing Company, Singapore, New Jersey, London and Hong Kong. https://doi.org/10.1142/3779

[12] Malkawi, E., Rousan, A., Rabei, E. and Widyan, H. (2002) Fractional Calculus and 
Applied Analysis, 5, 155.

[13] Agrawal, O.P. (1999) An Analytical Scheme for Stochastic Dynamics Systems Containing Fractional Derivatives. ASME Design Engineering Technical Conferences, (7), 243-250.

[14] Hasan, E.H. (2016) Applied Physics Research, 8, 60. https://doi.org/10.5539/apr.v8n3p60

[15] Jarab'ah, O. (2016) Science International Lahore, 28, 3365. http://www.sci-int.com/Search?catid=71

[16] Agrawal, O.P. (2001) Journal of Applied Mechanics, 68, 339. https://doi.org/10.1115/1.1352017

[17] Agrawal, O.P. (2002) Journal of Mathematical Analysis and Applications, 272, 368. https://doi.org/10.1016/S0022-247X(02)00180-4

[18] Igor, M., Sokolove, J.K. and Blumen, A. (2002) Physics Today, American Institute of Physics S-0031-9228-0211-030-1.

[19] Jarab'ah, O., Nawafleh, K. and Ghassib, H.B. (2013) European Scientific Journal, 9, 70. http://www.eujournal.org/index.php/esj/article/download/1946/1888 